\title{
Sleep Problems in Narcolepsy and the Role of Hypocretin/Orexin Deficiency
}

\author{
Emmanuel Mignot ${ }^{a} \quad$ Jamie Zeitzer ${ }^{a}$ Fabio Pizza ${ }^{b, c}$ \\ Giuseppe Plazzic, d
}

${ }^{a}$ Center for Narcolepsy, Stanford University, Palo Alto, CA, USA; ${ }^{b}$ Department of Biomedical and Neuromotor Sciences, University of Bologna, Bologna, Italy; ' IRCCS Istituto delle Scienze Neurologiche di Bologna,

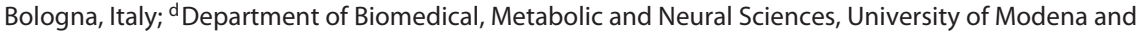
Reggio-Emilia, Modena, Italy

\begin{abstract}
Since its description in the 19th century, narcolepsy type 1 (NT1) has been considered as a model sleep disorder, and after the discovery of rapid eye movement (REM) sleep onset in the disorder, a gateway to understanding REM sleep. The discovery that NT1 is caused by hypocretin/orexin deficiency, together with neurochemical studies of this system, has helped to establish how this neuropeptide regulates the organization of sleep and wake in humans. Current analyses suggest that the main functions of the hypocretin/orexin system are (1) maintenance of wakefulness in the face of moderate sleep deprivation; (2) passive wake promotion, especially in the evening, driven by the circadian clock; (3) inhibition of REM sleep, with possible differential modulating effects on various subcomponents of the sleep-stage, explaining REM sleep dissociation events in NT1. Narcolepsy is also associated with an inability to consolidate sleep, a more complex phenotype that may result from secondary changes or be central to the role of hypocretin in coordinating the activity of other sleep- and wake-promoting systems. Novel technologies, such as the use of deep learning analysis of electroencephalographic signals, is revealing a complex pattern of sleep abnormalities in human narcolepsy that can be used diagnostically. The availability of novel devices measuring sleep $24 \mathrm{~h}$ per day also holds promise to provide new insights into how brain electrical activity and muscle tone are regulated by hypocretin.
\end{abstract}




\section{Introduction}

The study of sleep abnormalities in narcolepsy is grounded in its initial historical descriptions. Narcolepsy is a chronic neurological disorder that affects the ability to control sleep and wake states. It is characterized by excessive daytime sleepiness, cataplexy (sudden episodes of muscle weakness triggered by emotions), and other abnormal rapid eye movement (REM) sleep events, such as sleep paralysis (being paralyzed but awake at the same time), and hypnagogic hallucinations (dream-like experiences that occur while still conscious at sleep onset) [1].

It was only in the late 1970s, following the discovery that gamma hydroxybutyric acid (sodium oxybate) - a strong slow-wave sleep-enhancing agent - was an effective therapy on most narcolepsy symptoms [2], that disturbed nocturnal sleep became recognized as another cardinal symptom of narcolepsy. This classic presentation is currently referred to as narcolepsy type 1 (NT1) [3]. It is strongly associated with the immune gene Human Leukocyte Antigen (HLA)-DQB1*06:02 and, in most cases, is diagnosed in the presence of a positive Multiple Sleep Latency Test (MSLT) indicating rapid transitions to REM sleep. The cause of NT1 is an autoimmune-mediated loss of the cells producing the neuropeptide hypocretin (orexin) [4].

Although the term narcolepsy came later to also include cases without cataplexy (narcolepsy type 2 or NT2) but with a positive MSLT, the present chapter primarily concerns NT1, as patients with NT2 are mostly without hypocretin deficiency. We will only briefly discuss issues pertinent to NT2 toward the end of this chapter.

\section{The Effect of Acute Hypocretin/Orexin Cell Loss in Human Narcolepsy}

The acute effect of hypocretin/orexin cell loss is best assessed in NT1 children where the onset of symptoms is often extremely abrupt as it occurs within weeks. Pizza et al. [5] showed that narcolepsy first presents as a genuine hypersomnia with increased total daily sleep time over the 24-h day, occurrence of disturbing awakenings and dreaming, and prolonged daytime naps (sometimes lasting hours) (Fig. 1a). It is also associated with a generalized hypotonia with prominent facial involvement (mouth opening and tongue protrusion), a global floppy aspect, and gait disturbance in which falls to the ground can occur without apparent relation to emotions.

This phenotype suggests that the primary effect of hypocretin is promotion of wakefulness, inhibition of REM sleep occurrence, and regulation of muscle tone. Weight gain typically follows, abruptly in children [6], a phenotype likely to be complex and at least partially due to decreased activity and metabolism in the face of increased or similar appetite. Premature puberty may also ensue. Following this, children typically evolve toward the most well-known phenotype of adult narcolepsy, characterized by brief, refreshing sleep attacks, and episodes of muscle weakness evoked by strong emotions. Sleep becomes progressively more disrupted by wake transitions (Fig. 1b). Patients often report their 


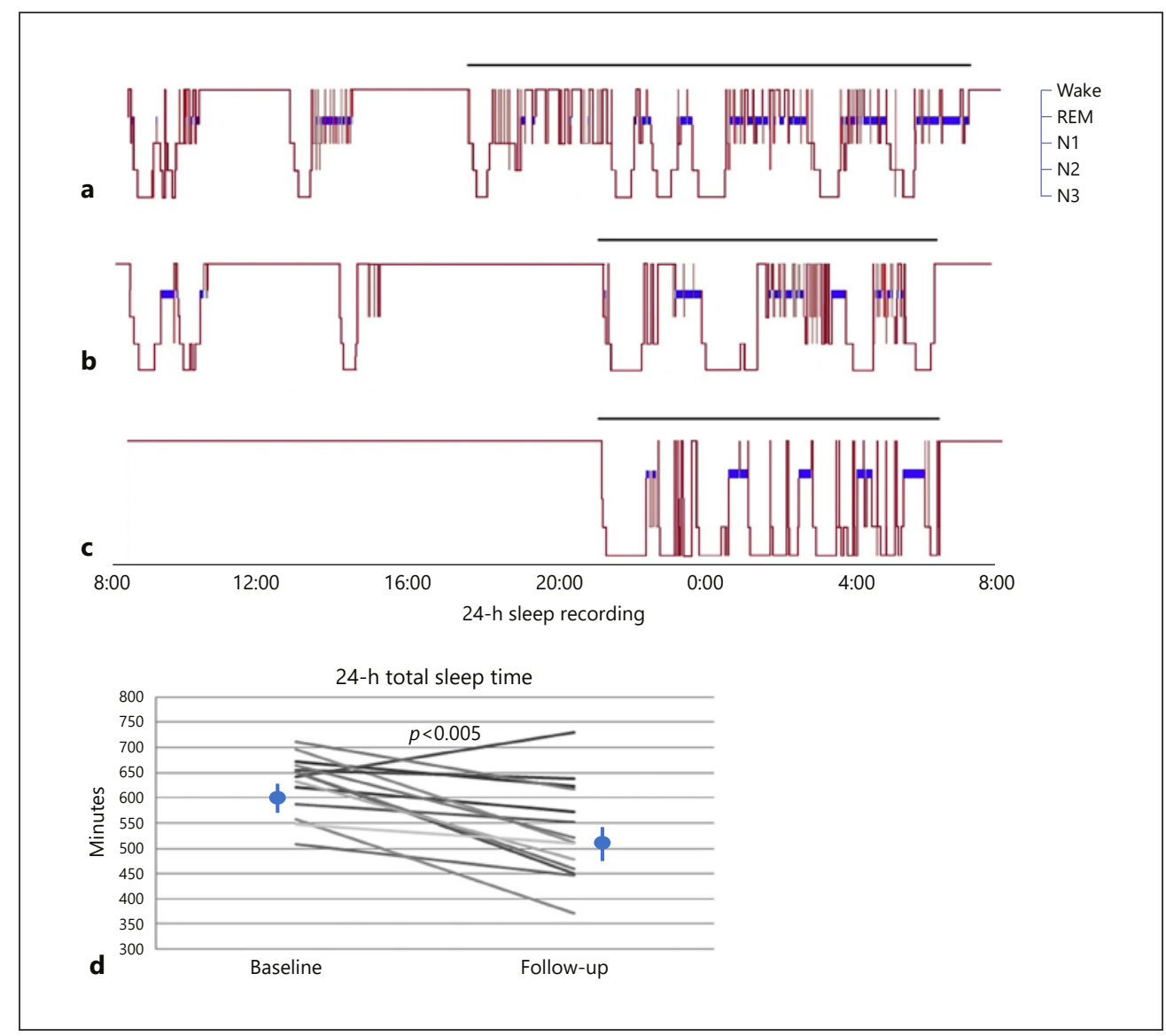

Fig. 1. Long-term (24-h) sleep recordings (split into wake, rapid eye movement [REM] sleep, and the three non-REM sleep phases, N1, N2, N3) in a patient suffering from narcolepsy type 1 (NT1) close to onset of disease (a), 6 months later (b), and in a control subject (c). After one day of acclimation, patients were allowed to sleep in free-running conditions, recorded by an ambulatory, wireless, device. Note fragmentation of sleep, wake, and REM sleep onset periods from wake all through the 24-h day in NT1, contrasting with normal wake during the day and sleep at night (black bar on top of the hypnogram) in the control. There is also increased total 24-h sleep time close to disease onset (a) and normal sleep time 3 months later (b) in patients with narcolepsy. A significant decrease in 24-h total sleep time is observed in these patients after a mean 3-year follow-up (d); for details, see Pizza et al. [5]. d Reproduced from Pizza et al. [5] with permission under the Creative Commons Attribution Non-Commercial 3.0 License (http://creativecommons.org/licenses/by-nc/3.0/).

sleep to be restless, a symptom improved by sodium oxybate, a drug that restores the proportion of deeper stages of sleep at night [7] and improves the entire phenotype. Cataplexy may also decrease with age, although this is now difficult to ascertain given the increase in the number of patients in whom cataplexy is adequately treated.

This is also in agreement with the effects of orexin antagonists, which promote sleep but also reduce REM sleep latency [8]. As orexin agonists are becoming available, a critical question of the field is whether these drugs will not only promote wakefulness and 
reduce cataplexy, which is preliminarily supported by pilot studies using the orexin receptor 2 agonist TAK925 (as presented at World Sleep 2019 in September 20-25, 2019, in Vancouver, Canada [9], and at the virtual European Sleep Research Society congress in September 22-24, 2020 [10]), but also normalize disrupted nocturnal sleep. Data to date suggest there are patients with narcolepsy who are hypersensitive to TAK925, with 2-3 times lower doses than in controls being wake-promoting (with dopaminergic stimulants, similar doses are generally needed to promote wakefulness in controls and narcoleptic patients $[11,12])$. Pharmacokinetic issues set aside, it remains to be seen if disturbed nocturnal sleep will resolve upon chronic administration of orexin receptor 2 agonists, or/and if dual orexin receptor agonism will be needed for full control of all narcolepsy symptoms.

\section{The Rise and Fall of the MSLT}

The MSLT was originally designed by Carskadon et al. [13] to measure sleep latency in relation to sleep deprivation. Mitler et al. [14] adapted the test to study narcolepsy by allowing longer naps to permit the observation of REM sleep onsets, defining a sleep onset REM period (SOREMP) as REM sleep latency $\leq 15 \mathrm{~min}$, and mean sleep latency $\leq 5 \mathrm{~min}$ and $\geq 2$ SOREMPs as diagnostic for the condition [13]. Indeed, authors had already shown that a SOREMP at night was highly characteristic of narcolepsy, but only occurred in half of cases (a SOREMP at night is very specific with only $0.8 \%$ false positive rate) $[15,16]$. Although the MSLT rapidly became the standard diagnostic test in the United States and World for narcolepsy, it was first characterized in very few controls. Only in the late 1990s were large numbers of patients without narcolepsy studied and it was discovered that there was a $4 \%$ false positive rate [17]. In the 2000s, studies were also performed in the general population showing a similar false positive rate [18], notably in association with shift work. This last finding is not surprising considering the strong gating of REM sleep by the circadian clock, with maximal propensity shortly after the trough of body temperature (e.g., 4-5 a.m.) [19]. Additional false positives were found in association with insufficient sleep [18]. To increase sensitivity, the threshold for sleep onset was later adjusted to a mean sleep latency $\leq 8 \mathrm{~min}$, maintaining the $\geq 2$ SOREMPs requirement. The diagnostic criteria were finally adjusted to include nocturnal sleep as valid for the $\geq 2$ SOREMPs required, although this change had minimal impact on the specificity or sensitivity of the MSLT in the determination of NT1.

The MSLT is a good test for diagnosing NT1, which is caused by hypocretin deficiency, with roughly $92 \%$ sensitivity and $96 \%$ specificity [16]. However, the potential overuse of the MSLT in patients with subjective sleepiness and no cataplexy has potentially created a large group of individuals misdiagnosed as having NT2; in these individuals, MSLT findings are non-repeatable in most cases, suggesting false positives. Further, a $4 \%$ false positive rate in $4-8 \%$ of the population (the rate of excessive daytime sleepiness in the general population) is $0.16-0.32 \%$, manyfold greater than the prevalence of NT1 with cataplexy $(0.03 \%)$ [20]. 
One issue with the MSLT may be the non-exclusion of chronic sleep deprivation, which is very difficult to demonstrate [21], even more so as actigraphy or sleep logs are not mandated prior to an MSLT, an addition that most experts believe should be mandatory. The non-reproducibility of the MSLT in NT2 [22] suggests that NT2 and idiopathic hypersomnia, now defined in the international classification as MSLT mean sleep latency $<8$ min and 0 or 1 SOREMP, should be merged [23]. This, however, could turn out to be problematic for many patients who rely on a NT2 diagnosis for drug coverage (idiopathic hypersomnia is often not covered by insurance), as it is clear that many of these patients, whether classified as idiopathic hypersomnia or NT2, can benefit from therapy. What the merged entity will be called will be the object of fierce debate, as the decision, which should be based on science alone, has serious consequences for patients, as insurance coverage leaves less and less room for patient-centered medicine. Future research needs to demonstrate that hypersomnolence syndromes without cataplexy are formally responsive to therapy.

\section{The Fall and Return of 24- to 48-h Continuous Sleep Recording Studies}

Prior to the MSLT, 24- or 48-h in-laboratory sleep recordings were conducted in narcolepsy patients to study the sleep patterns of these patients. These showed that established (i.e., long after onset of initial symptoms) NT1 was not a true hypersomnia. Indeed, these recordings revealed that most patients do not have increased 24-h total sleep time (unlike children close to onset, see above) but rather fragmented sleep and wake all through the $24 \mathrm{~h}$, with rapid transitions to REM sleep anytime during the 24-h day, a strong diagnostic feature [24]. Circadian rhythms of temperature and melatonin are intact, however. These results mirrored data by Carskadon and Dement [25], which asked control and narcolepsy subjects to live on a 90-min sleep/wake schedule ( $1 \mathrm{~h}$ of wake and $30 \mathrm{~min}$ of sleep opportunity) and found that during the 30 -min sleep opportunities, individuals with narcolepsy had REM transitions at all times during the day and night, while this occurred only in the early morning hours in controls (this remarkable study was the precursor of the MSLT [13]). These results, paralleling studies in animal models [26], showed that hypocretin activity inhibits REM sleep occurrence and that REM sleep propensity is at its highest when hypocretin levels are the lowest.

Considering the problems of the MSLT as currently performed, a return to long-term (24-72 h) sleep recordings has been advocated as a better option for diagnosing narcolepsy. Although by no means perfect to exclude the effect of poor sleep on a long-term basis, one advantage would be the ability to witness sleep rebound during the period of recording, should sleep deprivation be a major factor in a positive MSLT. Together with actigraphy, it is also likely to better exclude circadian disturbances, which can increase the likelihood of observing daytime SOREMPs in MSLTs [18]. Although less well characterized than the MSLT, selectivity and sensitivity of 24-72 h recordings are as high, or higher, for detecting NT1 [5, 24] (see Fig. 1a, b vs. c). 
The main issue regarding long-term sleep recordings is the difficulty and cost of implementing this in clinical practice, not to mention that measuring and detecting low levels of cerebrospinal fluid hypocretin-1 (orexin-A) is more definitive for diagnosing NT1 but more frequently used in Europe than in the US. For this reason, we recently advocated waiting for the advent of wearable polysomnographic (PSG)/EEG technology as these are likely to be developed in a few years [27]. These could be worn at home for multiple days and used to diagnose NT1 and validate sleep during self-reported sleep attacks or hypersomnia behavior. This approach, which was used as early as in 1988 in NT1 [28], would allow more objective differentiation with fatigue or clinophilia (the tendency of a patient to remain in bed in a reclined position without sleeping for prolonged periods) in depression, an important differential diagnosis in hypersomnia [29]. Such protocols would have more face validity and be more practical to conduct than 24 - to 48 -h in-laboratory studies. Although most experts agree this is the direction that needs to be taken, and there is no technical obstacle to creating the needed device, this is likely to take time because there is no business incentive to drive this agenda forward at the present time.

\section{Hypocretin/Orexin Release, Cell Activity, Sleep Function, and the Phenotype of Narcolepsy}

Another way to explore how hypocretin regulates sleep and wake is to study cell activity and hypocretin release across sleep-wake and various behaviors. Clearly, the function of hypocretin/orexin is unlikely to be solely the regulation of sleep; rather it is the only function that cannot be compensated when complete loss of the peptide occurs [30]. Nonetheless, as illustrated in Figure 2, numerous studies have shown that across species, extracellular hypocretin levels or release peak at the end of the active cycle (evening in humans) and decrease during sleep, to be minimal in the early morning hours in humans, not unlike core body temperature. This behavior is governed primarily by the circadian clock but also influenced by homeostatic pressure such that sleep deprivation increases hypocretin levels, while reducing sleep debt decreases levels [31-33].

This duality may be helpful in explaining some aspects of the phenotype of narcolepsy. For example, most (but not all) narcolepsy patients have no difficulties in falling asleep in the evening during the so-called "forbidden zone" (circadian time when temperature peaks, around 6-7 p.m.), suggesting that hypocretin is an important component of the suprachiasmatic nucleus-regulated wakefulness drive [25]. This is also supported by the observation that the hypocretin/orexin antagonist suvorexant increases total sleep time by more than $2 \mathrm{~h}$ in shift workers trying to sleep during the daytime [34]. Similarly, as hypocretin levels increase with sleep deprivation or sleep debt, it suggests that hypocretin may be an important system that must be recruited to counteract sleep deprivation, possibly in the context of motivated behaviors or emotions. This last function could explain why a major phenotype of narcolepsy is the inability to stay awake for long periods of times, with the need for naps every few hours to stay awake. 


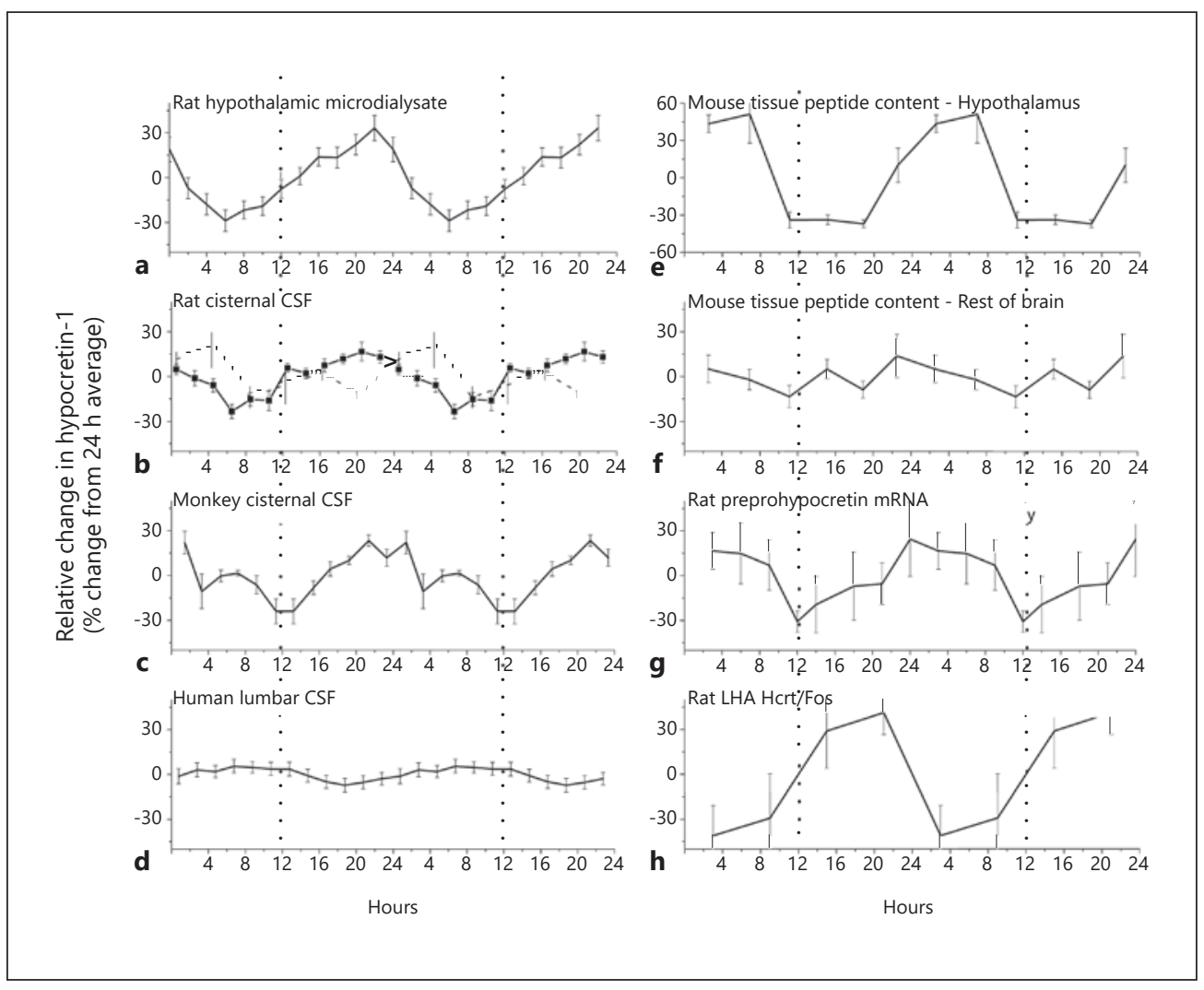

Fig. 2. Hypocretin-1 (orexin-A) peptide (a-f), preprohypocretin mRNA (g), and c-fos expression in hypocretin neurons, a marker of neuronal activity, (h) exhibit a robust daily variation across different species. Data have been normalized to the 24-h average and aligned according to time of activity onset (hour 12 , dotted line). Note the various sources and techniques used for assay. The dashed line in panel $b$ represents data from rats with lesioned suprachiasmatic nucleus. All data were collected while animals lived in a 12-h light:dark cycle. Data in a-f were obtained in our laboratory, and $\mathbf{g}$ and $\mathbf{h}$ were derived from graphically published data. Note the long delay and much reduced amplitude of hypocretin-1 fluctuations in human lumbar cerebrospinal fluid (CSF), reflecting an 8-h delay in CSF migration from the cisterna magna to the human lumbar sac; this allows measurement of CSF hypocretin-1 for the diagnosis of narcolepsy to be relatively insensitive to time of CSF draw. Reproduced from Zeitzer and Mignot [36] with permission of the Licensor through PLSclear.

In contrast to this picture, studies of neuronal activity, which are difficult to conduct as identification of the neuronal phenotype for each recorded cell is needed, have not been as enlightening, showing (in rodents) increased sudden bursts of activity during wakefulness, notably during goal directed behaviors [30]. This contrasts with a very high basal activity in brain slices when these neurons are deafferented, suggesting an important role of active inhibition in the regulation of hypocretin/orexin activity. How neuronal activity translates into hypocretin release at the level of the terminal area is however somewhat unclear. One study examined human hypocretin release in the hypothalamus and the 
amygdala and found it to be absent in sleep and increased in wakefulness, notably in the context of social interactions [35].

Hypocretin (hypocretin-1 and -2) content in the brain is very high, not only in the posterior hypothalamus where cell bodies are, but across a wide range of brain areas, indicating large storage of hypocretin-rich granules in terminal branches where release is possibly locally regulated [36]. This is also supported by the fact that hypocretin content in various brain areas, reflecting terminal stores, do not have the same phase as hypocretin levels when measured extracellularly (Fig. 2). Because of this and other findings, many of the effects of hypocretin/orexin have been suggested to be exerted by volume transmission (extracellular diffusion with release outside of synapses) [30]. This would explain why studies of hypocretin release versus cell activity do not match well.

Why a loss of hypocretin/orexin is associated with REM sleep abnormalities is also starting to be understood. Hypocretin release studies during REM sleep have not been performed and may be impossible to perform in specific brain areas. Nonetheless, hypocretin cells have complex projections from the hypothalamus to core brainstem sleep regulatory regions known to participate in muscle atonia regulation [30, 37, 38]. It is possible that hypocretin does not simply inhibit REM sleep, but has differential effects on specific REM sleep behaviors, such as atonia and dreaming, known to be regulated by slightly different brainstem nuclei.

Interestingly, REM sleep-specific deprivation induces sleepiness or rebound REM sleep (including sleep paralysis) but is unable to produce cataplexy. The genesis of cataplexy may therefore be more complex than that of other symptoms. One possibility is that cataplexy is a non-specific type of muscle weakness triggered by emotions that is normally compensated by hypocretin tone. Some authors have shown that even individuals without narcolepsy have subclinical atonia when laughing [39]. It may be that an additional challenge to muscle tone induced by emotion cannot be compensated in the face of a chronic absence of hypocretin. This would explain why, at the onset of narcolepsy, cataplexy manifests more as a generalized muscle weakness that occurs independent of emotions [5], and that it is only later in the course of the disease that the symptom evolves as typical cataplexy, with brief periods of muscle weakness triggered by emotions such as laughing. The general atonia would be compensated except for breakthrough episodes when emotionally challenged. Circuitry involving the amygdala is likely involved based on old studies in narcoleptic hypocretin receptor (HCRTR) 2 mutant canines [40] and more recent mouse orexin knockout work [41]. In support of this hypothesis, "cataplexy" triggered by laughing also occurs in other neurological disorders with central nervous system-mediated atonia but no hypocretin/orexin deficiency, such as Niemann Pick type C, Prader Willi [42], and, interestingly, Norrie's disease, a disease characterized by a loss of X-linked monoamine oxidase (MAO) genes (almost always associated with blindness) [43]. 


\section{Role of HCRTR1 and HCRTR2 Receptors in the Phenotype of Narcolepsy}

The hypocretin/orexin system mediates its action through two receptors, HCRT1 $\left(\mathrm{OX}_{1} \mathrm{R}\right)$ and HCRT2 $\left(\mathrm{OX}_{2} \mathrm{R}\right)$, which have complementary distributions in mammals [44]. HCRTR1 reacts to hypocretin-1 (orexin-A), and HCRTR2 reacts to both hypocretin-1 and 2 (orexin-B). Of notable interest is that these receptors are highly concentrated in monoaminergic neurons (among other locations), with HCRTR1 primarily located in the adrenergic locus coeruleus and HCRTR2 primarily located on histamine-producing neurons [30]. This is of interest considering the role of monoamines in wake and sleep regulation. Both receptors are primarily activating and primarily coupled to Gq. HCRTR2 is the more ancient family member, with HCRTR1 only appearing in mammals [45]. It is also the dominant receptor functionally, as HCRTR2 mutant animals display all the clinical hallmarks of narcolepsy in dogs and rodents (cataplexy and the inability to consolidate long wake bouts), while HCRTR1 knockout animals have only a mild sleep phenotype with increased REM sleep propensity [46]. Double HCRTR1/HCRTR2 knockout animals recapitulate the more severe phenotype of HCRT knockout animals, suggesting that HCRTR1 has compensatory effects in the presence of loss of HCRTR2 in addition to other functions [47]. Interestingly, although the phenotype of HCRTR1 or HCRTR2 loss-of-function mutations has not yet been described in humans, polymorphisms with an anticipated decreased function in both genes have significant effects on sleep symptoms, as reported in the UK Biobank [48, 49]. Such polymorphisms include rs2653349-A (I308V), in transmembrane domain 6 of HCRTR2 close to the binding site, which is associated with increased napping and morning preference, and rs2271933 (I408V, N terminal cytoplasmic tail) in HCRTR1 which is associated with increased napping $[48,49]$. These effects are consistent with the function of hypocretin/orexin proposed above.

\section{The Phenotype of Disturbed Nocturnal Sleep in Narcolepsy and Novel Methods of Evaluations}

As discussed above, the phenotype of narcolepsy, especially in established (non-recent onset) cases, includes disturbed nocturnal sleep. Disturbed nocturnal sleep in NT1 is a complex but disabling symptom that often deserves treatment on its own. Patients with narcolepsy typically have no problem falling asleep in the evening but will often awaken after 1-2 $\mathrm{h}$ and be unable to return to sleep. They often describe their sleep as restless and never deep, and although they feel refreshed after sleeping, sleep has felt very disrupted. Symptoms of abnormal REM sleep, such as hypnagogic hallucinations, half-conscious dream-like activity, sleep paralysis, sleep-talking, nightmares, and REM sleep behavior disorder may also manifest as intrinsic features $[50,51]$ and are reported as exhausting. Nocturnal PSG in established NT1 cases reveals a SOREMP in 40\% of cases [16], increased stage N1 (which, as described below, is likely a mixed state) [52], sleep fragmentation [53] with numerous awakenings lasting more than $2 \mathrm{~min}$ and often associated with direct REM 


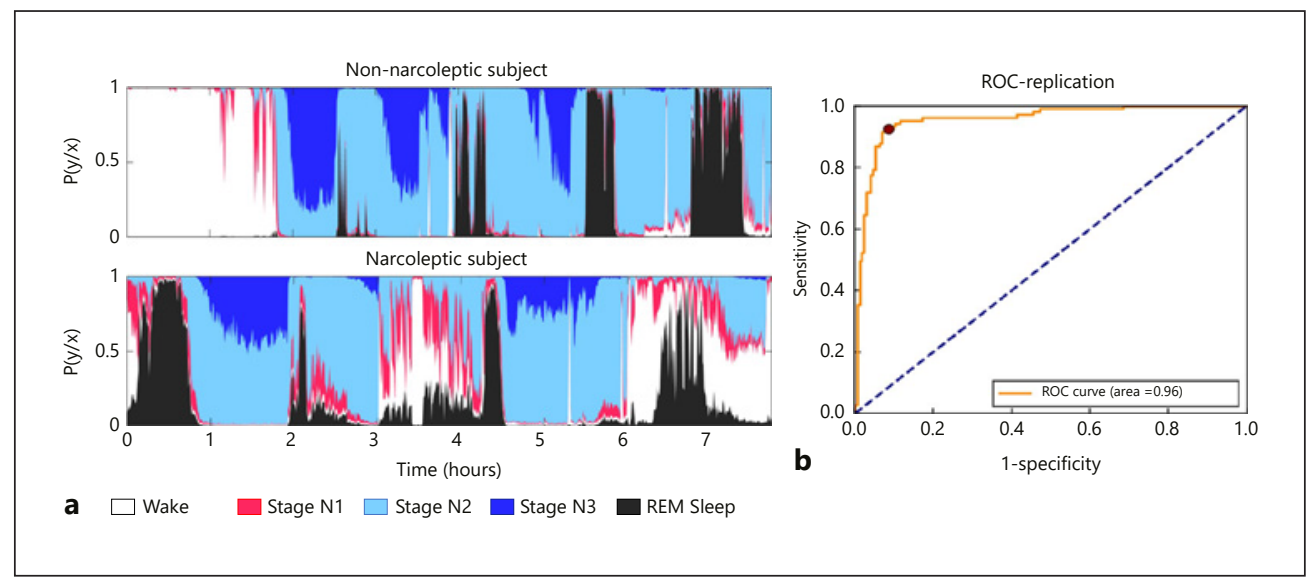

Fig. 3. a Abnormal sleep stage probabilities in narcolepsy type 1 (NT1) as determined using deep learning-based scoring of nocturnal polysomnography recordings. $\mathrm{P}(\mathrm{y} / \mathrm{x})$ represents probabilities of being in a specific sleep stage. Note short rapid eye movement (REM) sleep latency, sleep fragmentation, and sleep stage dissociation in narcolepsy, where periods of the hypnogram have equal probability of wake, REM sleep, and stage N1 (as often experienced by narcolepsy; may be sleep paralysis, hypnagogic hallucinations, etc.). $\mathbf{b}$ Features from the deep learning sleep scoring models were used to predict narcolepsy in a sample of untouched NT1 patients using a receiver operating curve (ROC) analysis, offering high diagnostic value. Modified from Stephansen et al. [56] with permission under the Creative Commons Attribution 4.0 International (CC BY 4.0) License (https://creativecommons.org/licenses/by/4.0/).

sleep transitions [54]. Interestingly, narcolepsy is also associated with increased periodic leg movements during sleep, and this forms part of the phenotype, as it is also associated with HLA-DQB1*06:02, a genetic marker of hypocretin deficiency in combined NT1/ NT2 samples [55].

Of notable importance, changes in macro-sleep architecture, such as a short REM sleep latency and abnormal numbers of sleep stage transitions, do not constitute the whole picture of sleep abnormalities in narcolepsy [53]. This is best exemplified by a study in our group that used deep learning to evaluate sleep stage characteristics of nocturnal sleep in untreated narcolepsy patients [56]. In this work, we used supervised deep learning to teach a neural network to score sleep stages every $30 \mathrm{~s}$ in controls, and in doing so, demonstrated that such an approach can be more accurate than human scoring. This neural network approach also allowed for the determination of the probability of each sleep stage during a 30-s epoch. That is, for a given 30-s epoch, what was the likelihood that the epoch was N1, N2, N3, or REM, with increasing probabilities being associated with a greater likelihood that the epoch is truly representative of that stage of sleep ("hypodensity plots," see Fig. 3). One reason that we used probabilities for each stage rather than just the categorical stage was the finding that sleep scoring accuracy did not deviate much among technicians across any sleep pathology except for narcolepsy, suggesting that characteristics of sleep stages in narcolepsy might be non-standard [56]. We therefore next used a second machine learning model, based on Gaussian process classifier that established a multipa- 
rameter value that was studied by receiver operating curve analysis (Fig. 3). This allowed for the selection of a threshold that could be used to diagnose NT1, a value that was independently validated in a separate group of NT1 patients. Using this methodology, we were able to show that PSG analysis of untreated narcolepsy patients was almost as predictive as the MSLT to diagnose narcolepsy. Not surprisingly, parameters selected as predictive included short REM sleep latency, increased number of sleep stage transitions, as well as presence of mixed probability sleep states, possibly representing sleep state dissociations, exemplar of the narcolepsy phenotype. Although our approach was successful, it only represents a first attempt at understanding sleep abnormalities in narcolepsy or in relation to decreased hypocretin tone. Indeed, narcolepsy predictions were estimated using a $30 \mathrm{~s}$ model of sleep scoring (a vestige of paper-based scoring rules) and did not take advantage of multiple EEG electrode locations (sleep-wake dissociation may also be regional, as reported for parasomnias, for example). Further, the machine learning model used was a simple long short-term memory recurrent neural network, and other models may improve our ability to differentiate this phenotype from other hypersomnias. We however also strongly believe these would be better tested when analyzing sleep and wake continuously for 24-48 h rather than during one night of sleep, and both at narcolepsy onset and in established cases. This would likely not only benefit NT1 diagnosis but also establish a better, data driven, classification for hypersomnia disorders.

\section{Key Take-Home Points}

- Hypocretin/orexin deficiency in NT1 is associated with complex sleep-wake abnormalities that change from onset to established cases.

- Hypocretin/orexin deficiency is best defined by a decreased wake drive and a disinhibition of REM sleep occurrence, which can occur as normal REM sleep or as wake-REM sleep dissociated events (sleep paralysis hallucinations, and maybe cataplexy). These disrupt both sleep and wake.

- Once established, disturbed nocturnal sleep in narcolepsy is best described as an inability to stay asleep for a long period of time accompanied by dissociated sleep stages such as vivid dreams with partial remembrance and consciousness, REM sleep behavior disorder and sleep paralysis.

- The peak of circadian alertness in the evening is likely blunted in NT1.

- The standard diagnostic test, the MSLT, although specific and sensitive for NT1, is now overused. It has lost its ability to diagnose hypocretin deficiency when cataplexy is not present. Better sleep recording techniques, such as continuous PSG recordings during 24-48 h need to better differentiate the cause of disorders of hypersomnolence. Novel sleep analytics, such as deep learning-based technology, are likely to play a critical role in the diagnosis of narcolepsy and other disorders with a centrally mediated hypersomnolence.

\section{Acknowledgments}

We thank all our colleagues and collaborators, as well as patients for their support. 


\section{Conflicts of Interest Statement}

E.M. received consultancy fees from Idorsia Pharmaceuticals Ltd during the development of this book. He also received consultancy fees from Merck and Eisai, and has been running clinical trials for Apple, Huami, Jazz Pharmaceuticals, and Sunovion. He also received research funding through Merck and Takeda. Finally, E.M. is on the advisory board and own stocks in Dreem, Orexia and Inoxia. J.Z., F.P. and G.P. have no conflicts of interest to declare.

\section{Funding Sources}

In the last 4 years, E.M. has mostly been funded by unrestricted gifts from patients, patient organization Wake Up Narcolepsy, and Jazz pharmaceuticals.

\section{Author Contributions}

All authors were involved in the development and review of the chapter, approved the final version to be published and take responsibility for all aspects of the work.

\section{References}

1 Daniels L: Narcolepsy. Medicine 1934;13:1-122.

2 Broughton R, Mamelak M: The treatment of narcolepsycataplexy with nocturnal gamma-hydroxybutyrate. Can J Neurol Sci 1979;6:1-6.

3 American Academy of Sleep Medicine: International Classification of Sleep Disorders - Third Edition (ICSD-

3). Darien, American Academy of Sleep Medicine, 2014

4 Nishino S, Ripley B, Overeem S, Lammers GJ, Mignot E: Hypocretin (orexin) deficiency in human narcolepsy. Lancet 2000;355:39-40.

5 Pizza F, Franceschini C, Peltola H, Vandi S, Finotti E, Ingravallo $\mathrm{F}$, et al: Clinical and polysomnographic course of childhood narcolepsy with cataplexy. Brain 2013;136(Pt 12):3787-3795.

6 Ponziani V, Gennari M, Pizza F, Balsamo A, Bernardi F, Plazzi G: Growing up with type 1 narcolepsy: its anthropometric and endocrine features. J Clin Sleep Med 2016; 12:1649-1657.

7 Black J, Pardi D, Hornfeldt CS, Inhaber N: The nightly administration of sodium oxybate results in significant reduction in the nocturnal sleep disruption of patients with narcolepsy. Sleep Med 2009;10:829-835.

8 Clark JW, Brian ML, Drummond SPA, Hoyer D, Jacobson LH: Effects of orexin receptor antagonism on human sleep architecture: a systematic review. Sleep Med Rev 2020;53:101332.

9 Evans R, Tanaka S, Tanaka S, Touno S, Shimizu K, Sakui $S$, et al: A Phase 1 single ascending dose study of a novel orexin 2 receptor agonist, TAK-925, in healthy volunteers (HV) and subjects with narcolepsy type 1 (NT1) to assess safety, tolerability, pharmacokinetics, and pharmacodynamic outcomes. Sleep Med 2019;64:S105-S106.
10 Tanaka S, Evans R, Alexander R, Imazaki M, Touno S, Shimizu K, et al: PO6. Selective orexin 2 receptor agonist TAK-925 to treat narcolepsy: results of a randomized, double-blind, placebo-controlled, multiple-ascending-dose, phase 1 study in patients with narcolepsy type 1. J Sleep Res 2020;29(S1):e13181.

11 Shelton J, Nishino S, Vaught J, Dement WC, Mignot E: Comparative effects of modafinil and amphetamine on daytime sleepiness and cataplexy of narcoleptic dogs. Sleep 1995;18:817-826.

12 Okuro M, Fujiki N, Kotorii N, Ishimaru Y, Sokoloff P, Nishino S: Effects of paraxanthine and caffeine on sleep, locomotor activity, and body temperature in orexin/ ataxin-3 transgenic narcoleptic mice. Sleep 2010;33: 930-942.

13 Carskadon MA, Dement WC, Mitler MM, Roth T, Westbrook PR, Keenan S: Guidelines for the multiple sleep latency test (MSLT): a standard measure of sleepiness. Sleep 1986;9:519-524.

14 Mitler MM, Van den Hoed J, Carskadon MA, Richardson G, Park R, Guilleminault C, et al: REM sleep episodes during the Multple Sleep Latency Test in narcoleptic patients. Electroencephalogr Clin Neurophysiol 1979;46:479-481.

15 Vogel G: Studies in psychophysiology of dreams III. The dream of narcolepsy. Arch Gen Psychiatry 1960;3:421428.

16 Andlauer O, Moore H, Jouhier L, Drake C, Peppard PE, Han F, et al: Nocturnal rapid eye movement sleep latency for identifying patients with narcolepsy/hypocretin deficiency. JAMA Neurol 2013;70:891-902. 
17 Aldrich MS, Chervin RD, Malow BA: Value of the multiple sleep latency test (MSLT) for the diagnosis of narcolepsy. Sleep 1997;20:620-629.

18 Goldbart A, Peppard P, Finn L, Ruoff CM, Barnet J, Young T, et al: Narcolepsy and predictors of positive MSLTs in the Wisconsin Sleep Cohort. Sleep 2014;37: 1043-1051.

19 Dijk DJ, Czeisler CA: Contribution of the circadian pacemaker and the sleep homeostat to sleep propensity, sleep structure, electroencephalographic slow waves, and sleep spindle activity in humans. J Neurosci 1995; 15(5 Pt 1):3526-3538.

20 Longstreth WT Jr, Koepsell TD, Ton TG, Hendrickson AF, van Belle G: The epidemiology of narcolepsy. Sleep 2007;30:13-26.

21 Baumann-Vogel H, Hoff S, Valko PO, Poryazova R, Werth E, Baumann CR: Extending sleep to confirm insufficient sleep syndrome is challenging. J Sleep Res 2020:e13109.

22 Ruoff C, Pizza F, Trotti LM, Sonka K, Vandi S, Cheung J, et al: The MSLT is repeatable in narcolepsy type 1 but not narcolepsy type 2: a retrospective patient study. J Clin Sleep Med 2018;14:65-74.

23 Fronczek R, Arnulf I, Baumann CR, Maski K, Pizza F, Trotti LM: To split or to lump? Classifying the central disorders of hypersomnolence. Sleep 2020;43:zsaa044.

24 Montplaisir J, Billiard M, Takahashi S, Bell IR, Guilleminault C, Dement WC: Twenty-four-hour recording in REM-narcoleptics with special reference to nocturnal sleep disruption. Biol Psychiatry 1978;13:73-89.

25 Carskadon MA, Dement WC: Sleep studies on a 90-minute day. Electroencephalogr Clin Neurophysiol 1975;39: 145-155.

26 Kantor S, Mochizuki T, Janisiewicz AM, Clark E, Nishino S, Scammell TE: Orexin neurons are necessary for the circadian control of REM sleep. Sleep 2009;32:11271134.

27 Maski K, Mignot E, Scammell TE: COMMENTARY on Lammers et al, Diagnosis of central disorders of hypersomnolence: challenges in defining central disorders of hypersomnolence. Sleep Med Rev 2020;52:101327.

28 Broughton R, Dunham W, Newman J, Lutley K, Duschesne P, Rivers M: Ambulatory $24 \mathrm{~h}$ sleep-wake monitoring in narcolepsy-cataplexy compared to matched controls. Electroencephalogr Clin Neurophysiol 1988;70:473-481.

29 Plante DT: Sleep propensity in psychiatric hypersomnolence: a systematic review and meta-analysis of multiple sleep latency test findings. Sleep Med Rev 2017;31:4857.

30 Nevarez N, de Lecea L: Recent advances in understanding the roles of hypocretin/orexin in arousal, affect, and motivation. F1000Res 2018;7:F1000 Faculty Rev-1421.

31 Yoshida Y, Fujiki N, Nakajima T, Ripley B, Matsumura $\mathrm{H}$, Yoneda $\mathrm{H}$, et al: Fluctuation of extracellular hypocre tin-1 (orexin A) levels in the rat in relation to the lightdark cycle and sleep-wake activities. Eur J Neurosci 2001;14:1075-1081.
32 Zeitzer JM, Buckmaster CL, Parker KJ, Hauck CM, Lyons DM, Mignot E: Circadian and homeostatic regulation of hypocretin in a primate model: implications for the consolidation of wakefulness. J Neurosci 2003;23: 3555-3560.

33 Zeitzer JM, Buckmaster CL, Landolt HP, Lyons DM, Mignot E: Modafinil and gamma-hydroxybutyrate have sleep state-specific pharmacological actions on hypocretin-1 physiology in a primate model of human sleep. Behav Pharmacol 2009;20:643-652.

34 Zeitzer JM, Joyce DS, McBean A, Quevedo YL, Hernandez B, Holty JE: Effect of suvorexant vs. placebo on total daytime sleep hours in shift workers: a randomized clinical trial. JAMA Netw Open 2020;3:e206614.

35 Blouin AM, Fried I, Wilson CL, Staba RJ, Behnke EJ, Lam HA, et al: Human hypocretin and melanin-concentrating hormone levels are linked to emotion and social interaction. Nat Commun 2013;4:1547.

36 Zeitzer J, Mignot E: Role of hypocretin/orexin in the neurobiology of sleep and alertness; in Bassetti C, Billiard M, Mignot E (eds): Narcolepsy and Hypersomnia. New York, Informa Healthcare, 2007, pp 359-374.

37 Lu J, Sherman D, Devor M, Saper CB: A putative flipflop switch for control of REM sleep. Nature 2006;441: 589-594.

38 Luppi PH, Fort P: Neuroanatomical and neurochemical bases of vigilance states. Handb Exp Pharmacol 2019; 253:35-58.

39 Overeem S, Reijntjes R, Huyser W, Lammers GJ, van Dijk JG: Corticospinal excitability during laughter: implications for cataplexy and the comparison with REM sleep atonia. J Sleep Res 2004;13:257-264.

40 Miller JD, Faull KF, Bowersox SS, Dement WC: CNS monoamines and their metabolites in canine narcolepsy: a replication study. Brain Res 1990;509:169-171.

41 Mahoney CE, Agostinelli LJ, Brooks JN, Lowell BB, Scammell TE: GABAergic neurons of the central amygdala promote cataplexy. J Neurosci 2017;37:3995-4006.

42 Mignot E, Lammers GJ, Ripley B, Okun M, Nevsimalova $\mathrm{S}$, Overeem S, et al: The role of cerebrospinal fluid hypocretin measurement in the diagnosis of narcolepsy and other hypersomnias. Arch Neurol 2002;59:1553-1562.

43 Koch H, Craig I, Dahlitz M, Denney R, Parkes D: Analysis of the monoamine oxidase genes and the Norrie disease gene locus in narcolepsy. Lancet 1999;353:645-646.

44 Marcus JN, Aschkenasi CJ, Lee CE, Chemelli RM, Saper $\mathrm{CB}$, Yanagisawa M, et al: Differential expression of orexin receptors 1 and 2 in the rat brain. J Comp Neurol 2001;435:6-25.

45 Cai Z, Liu H, Wang L, Li X, Bai L, Gan X, et al: Molecular evolutionary analysis of the HCRTR gene family in vertebrates. Biomed Res Int 2018;2018:8120263.

46 Mieda M, Hasegawa E, Kisanuki YY, Sinton CM, Yanagisawa M, Sakurai T: Differential roles of orexin receptor- 1 and -2 in the regulation of non-REM and REM sleep. J Neurosci 2011;31:6518-6526.

47 Willie JT, Chemelli RM, Sinton CM, Tokita S, Williams SC, Kisanuki YY, et al: Distinct narcolepsy syndromes in orexin receptor-2 and orexin null mice: molecular genetic dissection of non-REM and REM sleep regulatory processes. Neuron 2003;38:715-730. 
48 Kalmbach DA, Schneider LD, Cheung J, Bertrand SJ, Kariharan T, Pack AI, et al: Genetic basis of chronotype in humans: insights from three landmark GWAS. Sleep 2017;40:zsw048.

49 Wang H, Lane JM, Jones SE, Dashti HS, Ollila HM, Wood AR, et al: Genome-wide association analysis of self-reported daytime sleepiness identifies 42 loci that suggest biological subtypes. Nat Commun 2019;10:3503.

50 Antelmi E, Pizza F, Franceschini C, Ferri R, Plazzi G: REM sleep behavior disorder in narcolepsy: a secondary form or an intrinsic feature? Sleep Med Rev 2020;50: 101254.

51 Hishikawa Y, Kaneko Z: Electroencephalographic study on narcolepsy. Electroencephalogr Clin Neurophysiol 1965;18:249-259.

52 Roth T, Dauvilliers Y, Mignot E, Montplaisir J, Paul J, Swick T, et al: Disrupted nighttime sleep in narcolepsy. J Clin Sleep Med 2013;9:955-965.
53 Schoch SF, Werth E, Poryazova R, Scammell TE, Baumann CR, Imbach LL: Dysregulation of sleep behavioral states in narcolepsy. Sleep 2017;40:Zsx170.

54 Christensen JA, Carrillo O, Leary EB, Peppard PE, Young T, Sorensen HB, et al: Sleep-stage transitions during polysomnographic recordings as diagnostic features of type 1 narcolepsy. Sleep Med 2015;16:1558 1566.

55 Hong SC, Hayduk R, Lim J, Mignot E: Clinical and polysomnographic features in $\mathrm{DQB} 1{ }^{*} 0602$ positive and negative narcolepsy patients: results from the modafinil clinical trial. Sleep Med 2000;1:33-39.

56 Stephansen JB, Olesen AN, Olsen M, Ambati A, Leary EB, Moore HE, et al: Neural network analysis of sleep stages enables efficient diagnosis of narcolepsy. Nat Commun 2018;9:5229. 